\title{
Validation of a laboratory-constructed automated gas chromatograph for the measurement of ozone precursors through comparison with a commercial analogy
}

\author{
Jia-Lin Wang ${ }^{\mathrm{a}, *}$, Genn-Zhung Din ${ }^{\mathrm{a}}$, Chang-Chuan Chan ${ }^{\mathrm{b}}$ \\ ${ }^{a}$ Department of Chemistry, National Central University, Chungli 32054, Taiwan \\ ${ }^{\mathrm{b}}$ Institute of Occupational Medicine and Industrial Hygiene, National Taiwan University \\ College of Public Health, Taipei 100, Taiwan
}

\begin{abstract}
An automated gas chromatographic (auto-GC) system aiming at performing unattended hourly measurement of ozone precursors was developed in the laboratory. To encompass volatile organic compounds (VOCs) of a wide range of volatility within each analysis, the system uses dual-traps and dual-columns to simultaneously analyze both low and high-boiling compounds with each injection. Since sorbents with sufficient retention of $\mathrm{C}_{2}$ compounds at room temperature, namely ethane, ethene, and ethyne are not yet available, cooling with a thermoelectrical device was built around the low-boiling trap to facilitate quantitative enrichment of $\mathrm{C}_{2}$ compounds. The effectiveness of using micro-trap with low dead volume plumbing was manifested in reducing peak width and increasing peak height for particularly the lower-boiling compounds. The increase in sensitivity allowed sufficient detector response with a small amount of air sample, e.g. $200 \mathrm{ml}$ in our routine operation, which in term eliminate the need for remove water prior to sampling trapping. The performance and applicability of this laboratory-built auto-GC system was validated by comparison with a commercial analog, i.e. the ATD-400 system made by Perkin-Elmer, in the field sharing a common air intake. During more than 3 weeks of synchronized monitoring of ambient volatile organic compounds both systems showed highly consistent results on almost every monitored compound, clearly demonstrating the robustness of this self-built system. (C) 2003 Elsevier B.V. All rights reserved.
\end{abstract}

Keywords: Instrumentation; Air analysis; Environmental analysis; Volatile organic compounds; Hydrocarbons

\section{Introduction}

In accordance with the 1990 Clean Air Act Amendments [Section 182 (C) (1)], the US Environmental Protection Agency (EPA) developed rules for the initiation of photochemical assessment monitoring stations (PAMS) located in ozone non-attainment areas measuring a list of 56 non-methane hydrocarbons (NMHCs) [1,2]. Given the high complexity and low abundance nature associated with ambient volatile organic compounds (VOCs), the chromatographic methods are preferred for their efficient separation and detection capability over other analytical means [3-8].

In light of the deteriorating high ozone problem that plagues central and southern Taiwan. The Environmental Protection Agency of Taiwan recently started a VOC on-site

\footnotetext{
* Corresponding author. Fax: +886-34227972.

E-mail address: cwang@cc.ncu.edu.tw (J.-L. Wang).
}

monitoring network program in an attempt to explore the mechanism of ozone formation, which is unique to each non-attainment area. Each monitoring station houses an automated gas chromatographic (auto-GC) system (ATD-400, Perkin-Elmer) capable of measuring non-methane hydrocarbons from $\mathrm{C}_{2}$ to $\mathrm{C}_{12}$ of which more than 50 compounds are being targeted due primarily to their relatively high ambient concentrations and large ozone formation potentials. In addition to the commercial auto-GC systems used in the monitoring stations for continuous monitoring ozone precursors, a laboratory-built auto-GC system was also developed by us with similar capabilities to accommodate more versatile applications, e.g. canister analysis, mobile field deployment, validation of the station systems, etc.

The abundance of VOCs in ambient air can be highly variable and is in general in the range from several tens of $\mathrm{ppb}(\mathrm{v} / \mathrm{v})(\mathrm{ppbv})$ to $\mathrm{ppt}(\mathrm{v} / \mathrm{v})$ (pptv) levels. To determine trace amounts of atmospheric gases usually requires a concentration step as their concentrations are usually below 
the detection limits of most modern GC detection methods. This concentration step is usually achieved by freezing out gaseous constituents in an air sample using cryogens such as liquid nitrogen [8-14]. Furthermore, to efficiently resolve the large number of atmospheric constituents present in ambient air, it is generally essential to cryofocus the sample onto the front of a capillary column prior to the actual chromatographic separation. In remote monitoring stations the need of cryogen can present considerable running cost and logistical difficulties. To overcome these potential limitations, we have eliminated the dependency on liquid nitrogen by designing microtraps filled with carefully selected sorbents that can be cooled using commercially available refrigerated coolers or thermoelectric devices [15-21]. While commercial systems with cryogen free trapping or focusing features are available, the cost and flexibility often creates a barrier for budget-limited research laboratories. Thus, this work provides a simple recipe for constructing a cryogen-free preconcentration apparatus, which can be easily built and connected to any existing GC or GC-mass spectrometry (MS) in a laboratory with great flexibility and minimal cost as compared to employing a commercial auto-GC system.

\section{Instrumentation}

Instead of using the heart-cut technique to avoid high-boiling residual entering the porous-layer open tubular (PLOT) column as does the Perkin-Elmer system, this laboratory-constructed system used dual-traps and dual-columns to encompass the wide volatility range of components by employing one column for the gaseous compounds and the other for the higher boiling ones. A PLOT $\mathrm{Al}_{2} \mathrm{O}_{3} / \mathrm{KCl}$ column $(30 \mathrm{~m} \times 0.32 \mathrm{~mm}$; d.f. $=8 \mu \mathrm{m}$, Hewlett-Packard, San Fernando, CA, USA) was connected to one of the two sorbent traps for separating light VOCs. A DB- 1 column $(60 \mathrm{~m} \times 0.32 \mathrm{~mm}$; d.f. $=1.0 \mu \mathrm{m}, \mathrm{J} \& \mathrm{~W}$ Scientific, Folsom, CA, USA) was connected to the other trap for separating $\mathrm{C}_{6}-\mathrm{C}_{12}$ compounds. Different combinations of sorbents were used for the two traps with the trap for the PLOT column packed with Carbosieve SIII and Carboxen 1000 to retain mainly the smaller molecules, while the trap for the DB-1 column was packed with Carboxen 1000, Carboxen 1003 and Carbotrap to mainly retain high-boiling molecules. Packing of the trap was done by filling each sorbent to $1-2 \mathrm{~cm}$ within a piece of $3.2 \mathrm{~mm}$ o.d. stainless steel tubing with glass wool plugs placed in between and at both ends.

During desorption the trap for the PLOT column was ramped from -30 to $250^{\circ} \mathrm{C}$ within $7 \mathrm{~s}$, whereas the trap for the DB- 1 column was ramped from 30 to $280^{\circ} \mathrm{C}$. Meanwhile the flow of injection was reversed to backflush the retained VOCs to the columns for separation. After $5 \mathrm{~min}$ both traps were raised to $300^{\circ} \mathrm{C}$ to bake out the residual of the traps. Fig. 1 shows the valve configuration for the dual-trap dual-column system. The system was tested for the build-up of high-boiling residual, which would result into a gradual rise in baseline level and ghost peaks [22,23]. On numerous

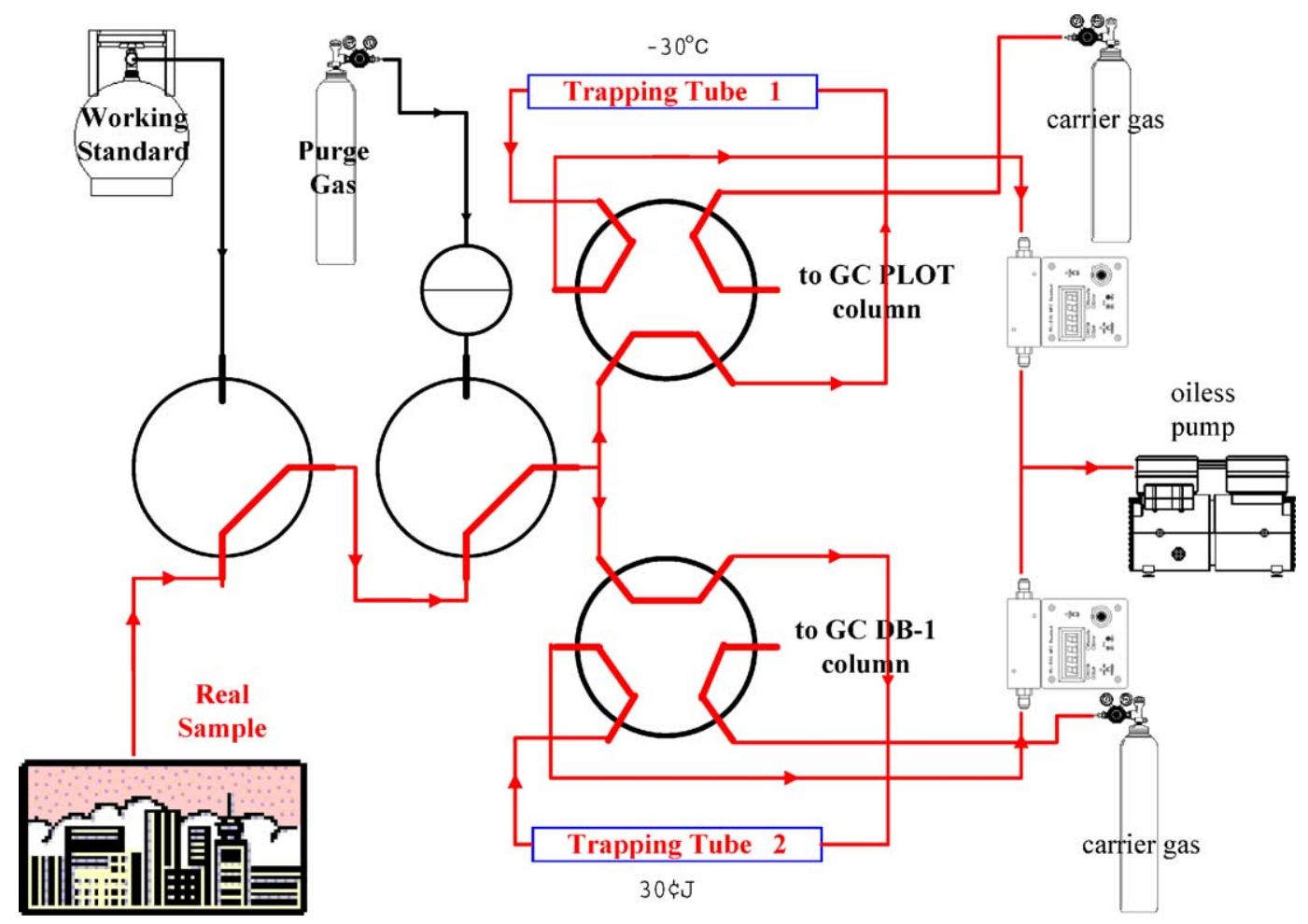

Fig. 1. Schematic diagram for the valve configuration of the dual-trap and dual-column auto-GC system. Valve switching positions shown in this figure correspond to the trapping mode in which the traps for the PLOT and DB- 1 columns are kept at -30 and $30^{\circ} \mathrm{C}$, respectively. 
occasions hundreds of continuous runs were performed in the laboratory or field and no sign of accumulation occurred.

Control software was also designed with self-explanatory icons so that the operator can have an easy grasp of the analytical status. Monitoring data were downloaded to the main laboratory on a daily basis over the Internet.

\section{Enrichment of extremely volatile compounds}

Because up until now no commercial sorbents can provide strong retention for extremely volatile VOCs such as the $\mathrm{C}_{2}$ hydrocarbons, i.e. ethane, ethene, and ethyne, at room temperature due to insufficient attraction, presumably the Van der Waals forces, within the micropores of sorbent media, reducing trapping temperature is necessary to decrease the mobility of these gaseous molecules. Thus, the trap design had to incorporate a cooling mechanism in addition to the rapid heating mechanism for thermal desorption. As a result, a retractable platform was built from a gas-actuated cylinder, on which the trap was fixed at one end of the cylinder's shaft, and the cooling block was placed at the opposite end (see Fig. 2). The entire assembly is housed in a $20 \mathrm{~cm} \times 15 \mathrm{~cm} \times 10 \mathrm{~cm}$ sealed case constantly purged by a flow of dry air $(150 \mathrm{ml} / \mathrm{min})$ coming from the zero air generator to prevent water vapor condensing onto the thermoeletric cooler (TEC) and ultimately affects the cooling efficiency. The cooling block consists of a thin copper plate (1-2 mm in thickness) bound to the cold side of a $40 \mathrm{~mm} \times 40 \mathrm{~mm}$ two stage TEC, the working of which was based on the Peltier effect, and a water-cooled radiator adhered to the hot side to serve as the heat sink. The square copper plate was machined to form a notch, onto which the $1 / 8 \mathrm{in} .(1 \mathrm{in} .=2.54 \mathrm{~cm})$ trap tubing can tightly recess when engaging.

When cooling is required the trap is pushed against the cooling block whose temperature has been maintained at $-30^{\circ} \mathrm{C}$. Once the enrichment is complete, the trap is pulled away from the block and is immediately heated up to $250{ }^{\circ} \mathrm{C}$ within $7 \mathrm{~s}$ to thermally desorb VOCs onto the column. Heating of the trap was made possible by supplying a low-voltage large current ( $4 \mathrm{~V} \mathrm{ac}$; $40 \mathrm{~A}$ ) to the stainless steel trap tubing, which enabled a maximum heating rate of $50^{\circ} \mathrm{C} / \mathrm{s}$ to its high temperature set point of $250^{\circ} \mathrm{C}$ for injection, and ultimately to $300^{\circ} \mathrm{C}$ for trap cleaning.

While separation is undergoing and the trap cleaning has been completed, the power to the trap tubing is turned off and the trap is pushed against the TEC unit to be cooled down for trapping the next sample aliquot.

Water was not removed by any physical or chemical means, such as the Nafion tubing or drying agents, during trapping to avoid possible artifacts incurred by the technique itself. As a result, plugging of the trap by water at $-30{ }^{\circ} \mathrm{C}$ was a major concern for the PLOT trap. Tests were made by drawing air of high humidity to determine the safe limit of trapping volume for the PLOT trap, which was determined as $250 \mathrm{ml}$ at the relative humidity of close to $100 \%$. Water plugging was never a concern for the DB-1 trap as trapping with hydrophobic sorbents at $30^{\circ} \mathrm{C}$ was way above the dew point of ambient air. As a result, more air can be drawn through the DB-1 trap to increase sensitivity, but in this experiment same air volume (approximately $200 \mathrm{ml}$ ) was enriched on both traps.

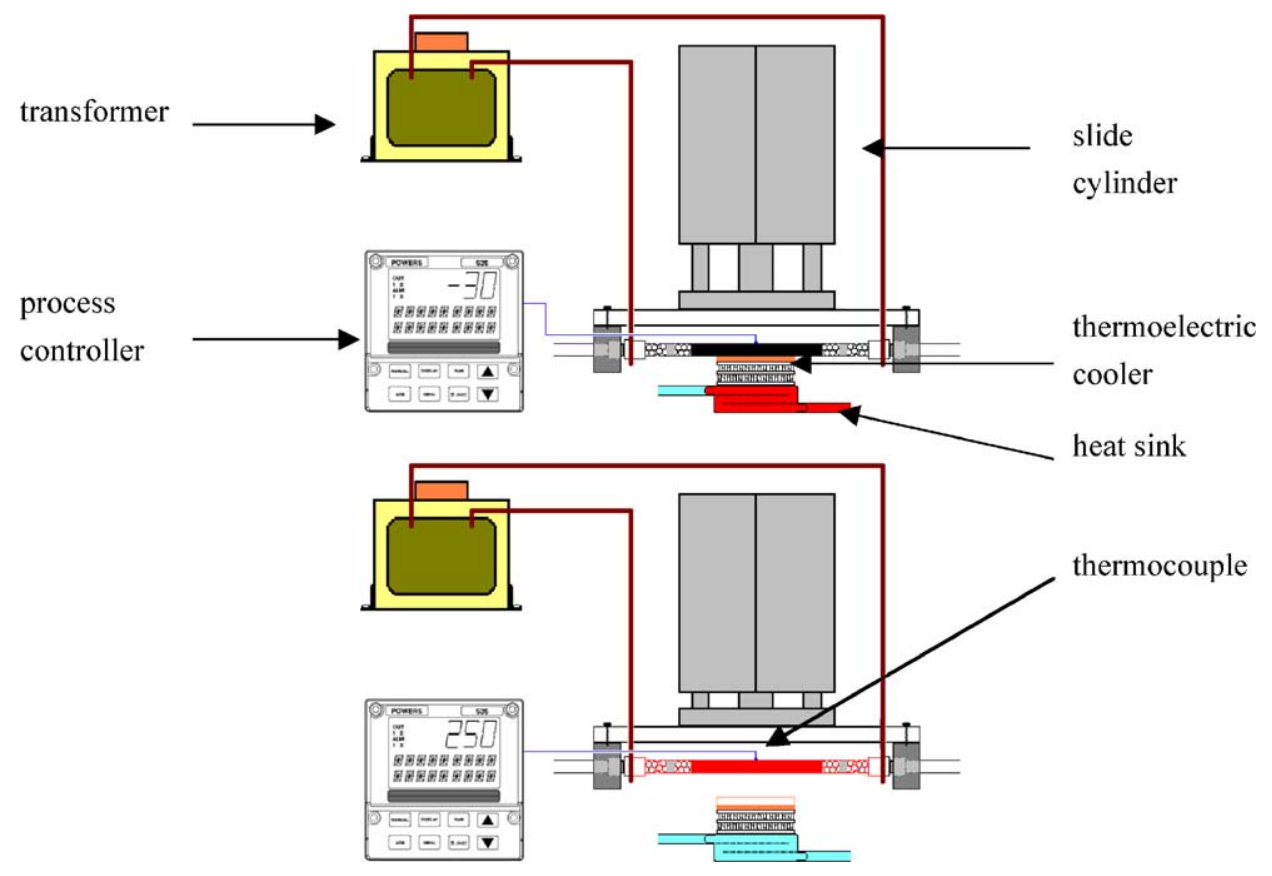

Fig. 2. A retractable device built for fast cooling and heating of the trap for PLOT column. In the upper figure the trap is pushed against the thermoelectric cooler by a pneumatic slide cylinder to freeze it to $-30^{\circ} \mathrm{C}$ for the enrichment of low-boiling VOCs. Upon thermal desorption the trap is pulled away from the TEC and heated up to $250^{\circ} \mathrm{C}$, see bottom figure. 


\section{System's performance on reproducibility and linearity}

Measurement precision was examined by repeatedly injecting a diluted standard mixture prepared at about $30 \mathrm{ppbv}$, see Fig. 3 for the chromatograms from the PLOT and DB-1 columns. Species identification was performed by connect- ing the preconcentrator to a GC-MS system (HP6890/5973) separately for the PLOT and DB-1 columns. Analytical precisions for selected NMHCs are listed in Table 1. In general, the precision for the $\mathrm{C}_{2}-\mathrm{C}_{12}$ NMHCs were usually better than $2 \%$. Because two traps were used with each connected to a particular column for optimal separation of a range of NMHCs, compounds in the $\mathrm{C}_{6}$ range, e.g. benzene, can be

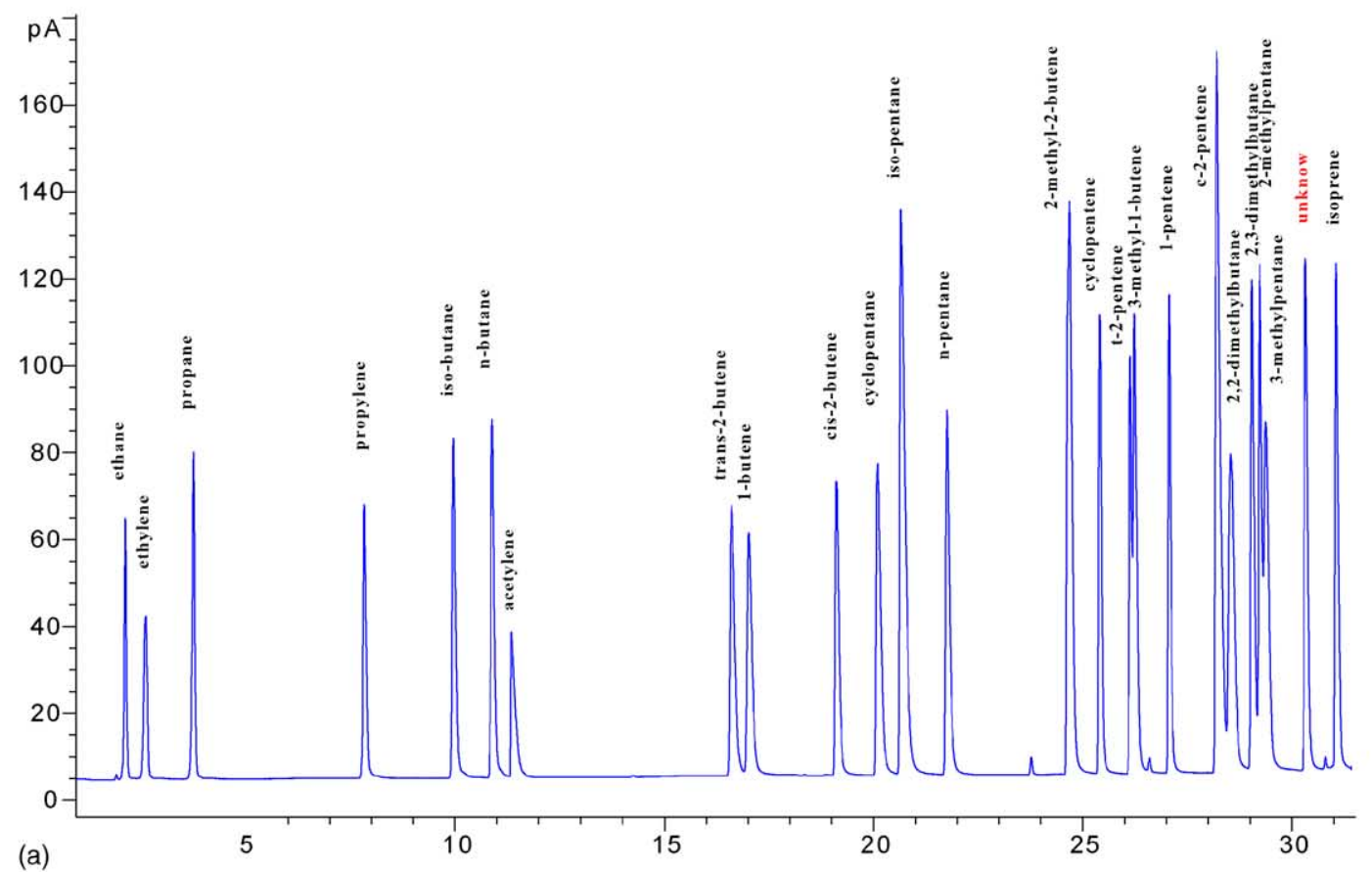

(a)



Fig. 3. (a) Analysis of a standard gas mixture for $\mathrm{C}_{2}-\mathrm{C}_{6}$ non-methane hydrocarbons on the PLOT column. (b) Analysis of a standard gas mixture for $\mathrm{C}_{6}-\mathrm{C}_{12}$ non-methane hydrocarbons on the DB-1 column. 
trapped by both traps and thus eluted from both columns (see Table 1). Since compounds such as benzene can be seen from either column, the precision of benzene on both columns ought to be rather close, which offers a merit for system diagnosis and can signal faulty measurement if the trend of benzene from the two columns disagree with each other.

Linearity and the breakthrough was tested by preparing a series of standard mixtures of $5,10,15,20,30,50$ ppbv con- centrations stored in 61 canisters. Out of the 56 target compounds, more than 46 compounds exhibited excellent linearity with the correlation coefficients better than 0.995 . The $R^{2}$ is generally poorer for the less volatile compounds with carbon number larger than 9, such as propyl benzene and 1,3,5-trimethylbenzene, were slightly poorer but still better than 0.980 . We suspect that surface adsorption occurred inside the canisters, which although claimed to be surface
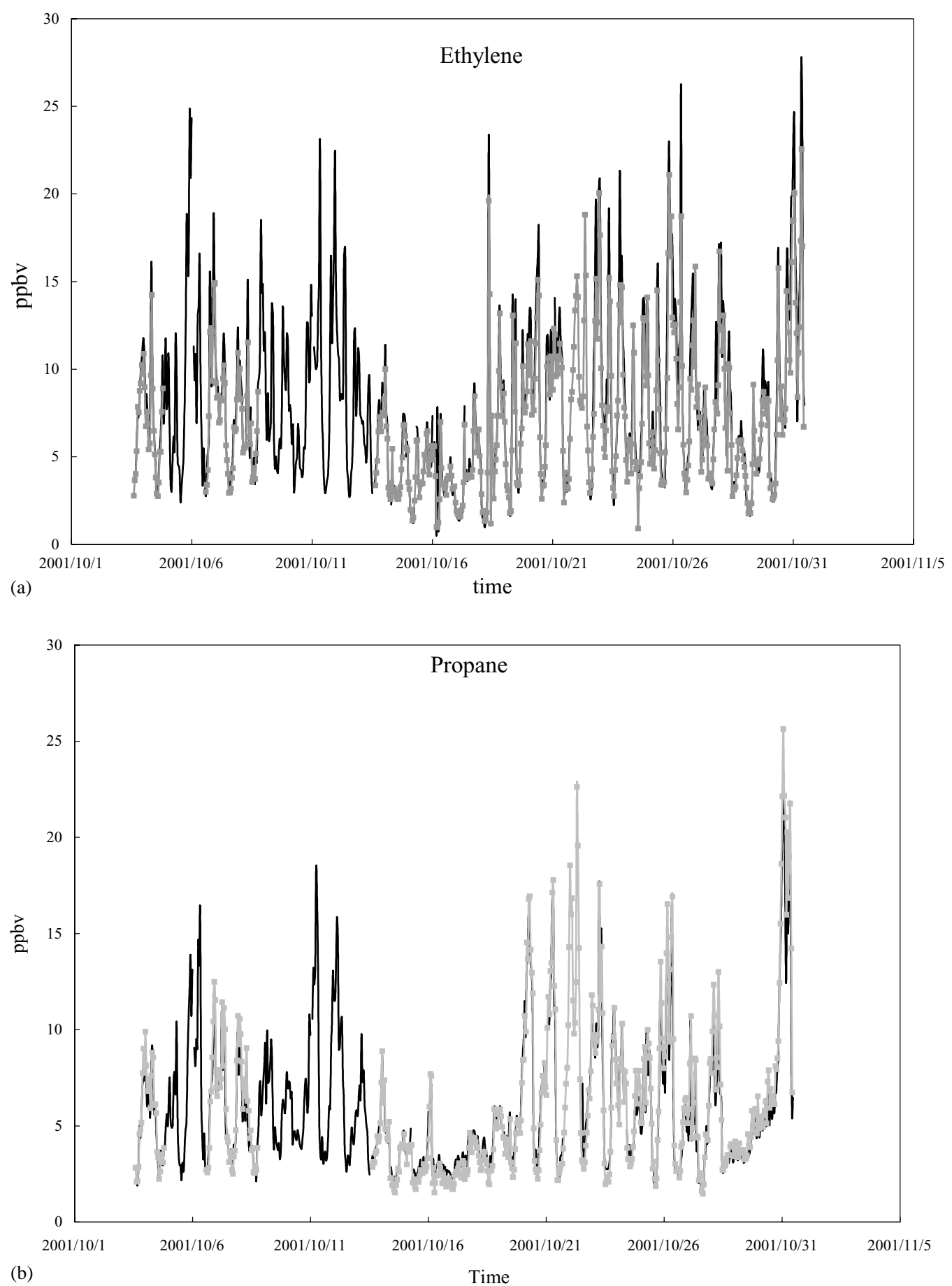

Fig. 4. (a) Comparison of ethene between a commercial auto-GC system shown by the gray dotted line and the laboratory-made system shown by the dark line. Each data point denotes an hourly measurement. (b) Comparison of propane. (c) Comparison of isoprene. (d) Comparison of benzene. 

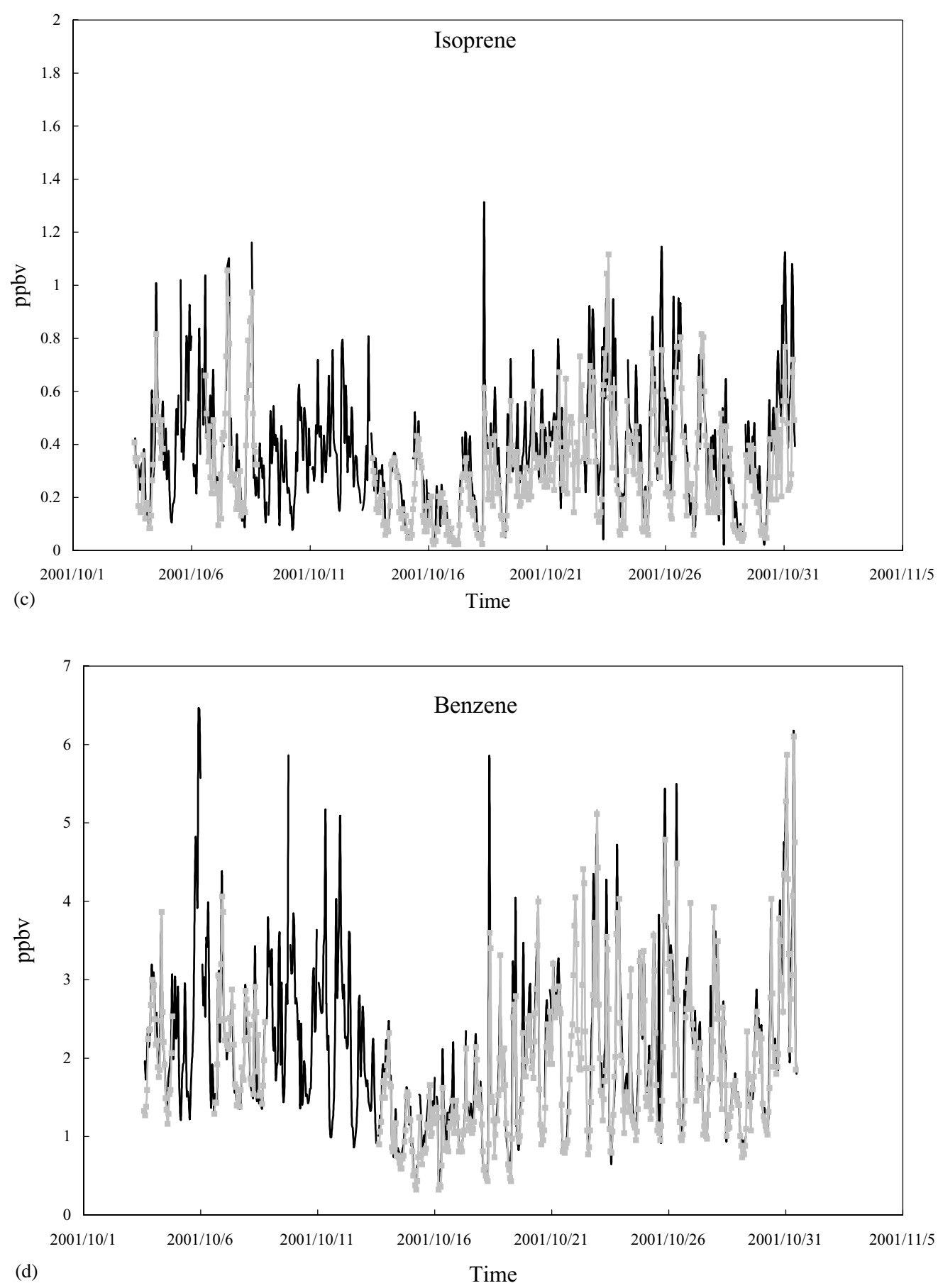

Fig. 4. (Continued).

treated, inevitably affected the less volatile compounds during storage and transfer, resulting in poorer reproducibility and linearity [24]. While the precision of $C_{3}$ or larger molecules can be quantitatively trapped at room temperature and thus easily show satisfactory precision and linearity, the trapping for $\mathrm{C}_{2}$ compounds, i.e. ethane, ethene, and ethyne, however requires sub-ambient temperature trapping and are highly subject to precise temperature control and mechanical stability. Because linearity can extend up to $50 \mathrm{ppbv}$ and beyond, which is way above what our urban station had been experienced, the trapping breakthrough was not a concern.

\section{Inter-system comparison}

Both the commercial and laboratory-built systems were housed in a trailer sharing a common air inlet made of Pyrex 
Table 1

Analytical precision for selected NMHCs eluted from two separated columns, i.e. PLOT and DB-1, based on eight replicates of a diluted standard mixture with $\mathrm{NMHC}$ concentrations at $30 \mathrm{ppbv}$

\begin{tabular}{|c|c|c|c|}
\hline \multicolumn{2}{|l|}{ PLOT } & \multicolumn{2}{|l|}{ DB-1 } \\
\hline Compound & $\begin{array}{l}\text { R.S.D. } \\
(\%)\end{array}$ & Compound & $\begin{array}{l}\text { R.S.D. }(\%) \\
(n=8)\end{array}$ \\
\hline Ethane & 0.80 & Hexane & 1.95 \\
\hline Ethene & 0.82 & Benzene & 2.06 \\
\hline Propane & 1.07 & cis-2-Hexene & 1.86 \\
\hline Propene & 1.10 & 2,4-Dimethylpentane & 1.46 \\
\hline Isobutane & 1.21 & Benzene & 2.06 \\
\hline$n$-Butane & 1.19 & 2,3-Dimethylpentane & 1.62 \\
\hline Ethyne & 1.31 & 3-Methylhexane & 1.56 \\
\hline trans-2-Butene & 0.94 & 2,3,4-Trimethylpentane & 1.24 \\
\hline 1-Butene & 1.15 & Heptane & 1.48 \\
\hline cis-2-Butene & 1.23 & Methylcyclohexane & 1.61 \\
\hline Cyclopentane & 1.25 & Toluene & 0.91 \\
\hline 2-Methyl-2-butene & 4.45 & 2-Methylheptane & 1.14 \\
\hline$n$-Pentane & 1.42 & 3-Methylheptane & 1.11 \\
\hline Cyclopentene & 2.26 & Octane & 1.09 \\
\hline 3-Methyl-1-butene & 1.39 & Ethylbenzene & 1.18 \\
\hline 1-Pentene & 1.32 & Styrene & 1.06 \\
\hline trans-2-Pentene & 2.08 & $m, p$-Xylenes & 1.46 \\
\hline 2,2-Dimethylbutane & 1.63 & $o$-Xylene & 1.48 \\
\hline 2,3-Dimethylbutane & 2.59 & Nonane & 1.48 \\
\hline Isoprene & 1.22 & $n$-Propylbenzene & 1.48 \\
\hline Hexane & 1.489 & Isopylbenzene & 1.90 \\
\hline Benzene & 0.988 & 1,3,5-Trimethylbenzene & 1.75 \\
\hline
\end{tabular}

glass. The trailer was placed in a public park surrounded by major roads and apartment buildings. Both traffic and household emissions were the dominant sources of VOCs, which is typical for an urban environment in Taiwan. Data from both systems were downloaded on daily basis and processed in the laboratory about $200 \mathrm{~km}$ from the station. Although both systems performed hourly continuous measurement, the sampling duration and starting time were different. For each hourly analysis the Perkin-Elmer system sampled ambient air for $45 \mathrm{~min}$ at $15 \mathrm{ml} / \mathrm{min}$, whereas our laboratory-built system sampled air for $10 \mathrm{~min}$ at $20 \mathrm{ml} / \mathrm{min}$. As a result, a slight discrepancy in the reported concentrations between the two systems was expected, since ambient VOC concentrations could be highly variable with respect to time and space.

Calibration was performed on-site to determine the response factor for each compound on both systems, so that the observed concentrations from the two systems can be directly compared on the same scale. Four standard mixtures were prepared in the laboratory and brought to the station for constructing calibration curves for the 56 NMHCs. Synchronized hourly measurement of the two systems was then started on 3 October 2001 and ended on 11 November 2001. Except two incidents of malfunction occurred with the PE system resulting in the loss of data in two time periods, a total of more than 300 continuous hourly measurements could be compared, see Fig. 4 for four compounds, i.e. ethene, propane, isoprene, and benzene, across a wide range of volatility, which were representative of a large array of monitored ozone precursors. Moreover, the selection of these four compounds had specific analytical purposes. For instance, ethene is one of the three $\mathrm{C}_{2}$ species, i.e. ethane, ethene, and ethyne, which can only be quantitatively trapped at reduced temperatures and the reliability of the cooling device controlled the quality of the $\mathrm{C}_{2}$ measurement. Propane, on the other hand, is the most volatile VOC second to the $\mathrm{C}_{2}$ species and can be quantitatively enriched without cooling. Cooling the trap dramatically increased the breakthrough volume of propane. Compounds with higher volatility than propane showed less or little gain in sensitivity via chilling. As a result, inconsistent trapping conditions arising from poor cooling device construction could easily manifest on the propane's result. Consequently we compared propane's precision between cooling and non-cooling conditions for inspecting the performance of the cooling device. Isoprene was chosen because it was the last monitored species on the PLOT column and it is the most important ozone precursor of biogenic origin in terms of ozone production potential $[25,26]$. To assess the performance of the other trap and DB-1 column, benzene is chosen due to its pervasive existence in ambient air and that it is one of the most concerned toxic pollutants in an urban environment [27]. Despite the mismatch in the sampling start-up time and duration for each injection, the month-long comparison in general showed rather consistent results between the two systems. The very fine details in the abrupt changes in concentration were also captured by both systems.

\section{Conclusion}

The objective of this research was to build an automated GC system for unattended continuous monitoring ozone precursors from $\mathrm{C}_{2}$ to $\mathrm{C}_{12}$ at ambient concentrations. To encompass a wide range of compounds of large differences in volatility, dual-traps and dual-columns were employed with the PLOT column designated for low-boiling compounds $\mathrm{C}_{2}-\mathrm{C}_{6}$ and the DB-1 column for $\mathrm{C}_{6}-\mathrm{C}_{12}$. A retractable cooling device made from a thermoelectric cooler was constructed to lower the cooling temperature to $-30^{\circ} \mathrm{C}$ to increase the retention of the three extremely volatile $\mathrm{C}_{2}$ species, namely ethane, ethene, and ethyne. Validation was conducted in the laboratory from the aspects of linearity and precision. In addition, the laboratory-made system was brought to the field and compared with a commercial GC system made by Perkin-Elmer which has been used in large quantity in the US PAMS program. In a month-long parallel comparison, excellent agreement in reported concentration levels between the two systems was observed. By reference to a commercial system the performance of the laboratory-made system was adequately validated and, therefore, this work provides a simple recipe for a budget-concerned laboratory to make a system of their own at minimal cost. 


\section{Acknowledgements}

This research was supported by the National Science Council of Taiwan under the contract number NSC91-2113M008021. The authors would like to thank the Environmental Protection Agency of Taiwan for allowing the study to be conducted in a photochemical assessment monitoring station.

\section{References}

[1] A.R. MacKensie, R.M. Harrison, I. Colbeck, C.N. Hewitt, Atmos. Environ. 25A (1991) 351.

[2] http://www.epa.gov/oar/oaqps/pams/.

[3] T. Maeda, S. Onodera, H. Ogino, J. Chromatogr. A 710 (1995) 51.

[4] J.F. Pankow, W. Luo, L.M. Isabelle, D.A. Bender, R.J. Baker, Anal. Chem. 70 (1998) 5213.

[5] D. Helming, J. Chromatogr. A 843 (1999) 129.

[6] J.L. Wang, W.L. Chen, Y.H. Lin, C.H. Tasi, J. Chromatogr. A 896 (2000) 31

[7] C.C. Chung, G.G. Lo, C.H. Tsai, J.L. Wang, Environ. Sci. Technol. 35 (2001) 3273

[8] J.M. Sanchez, R.D. Sacks, Anal. Chem. 75 (2003) 978

[9] A.H. Goldstein, B.C. Daube, J.W. Munger, S.C. Wofsy, J. Atmos. Chem. 21 (1995) 43.

[10] J.P. Greenberg, D. Helming, P.R. Zimmerman, J. Geophys. Res. 101 (1996) 14581.
[11] D. Klemp, D. Kley, F. Kramp, H.J. Buers, G. Pilwat, F. Flock, H.W Patz, A. Volz-Thomas, J. Atmos. Chem. 28 (1997) 135.

[12] L.M. Cardenas, J.F. Austin, R.A. Burgess, K.C. Clemitshaw, S. Dorling, S.A. Penkett, R.M. Harrison, Atmos. Environ. 32 (1998) 3339.

[13] M.H. Habram, J. Slemr, T. Welsch, J. High Resolut. Chromatogr. 21 (1998) 209

[14] J.-L. Wang, C.J. Chang, W.D. Chang, C. Chew, S.W. Chen, J. Chromatogr. A 844 (1999) 259.

[15] M.R. Bassford, P.G. Simmonds, G. Nickless, Anal. Chem. 70 (1998) 958.

[16] E.E. Ferguson, Summary Report No. 20, Climate Monitoring and Diagnostics Laboratory, 1991.

[17] W.A. McClenny, J.D. Pleil, K.D. Oliver, J. Air Pollut. Control Assoc. 37 (1987) 244

[18] E. Matisova, S. Skrabakova, J. Chromatogr. A 707 (1995) 145.

[19] K. Oliver, J.R. Adams, E.H. Daughtrey Jr., W.A. Mcclenny, M.J. Yoong, Atmos. Environ. 30 (1996) 2751.

[20] M. Harper, J. Chromatogr. A 885 (2000) 129.

[21] M. Holdren, D. Danhof, M. Grassi, J. Stets, B. Keigley, V. Woodruff, A. Scrugli, Anal. Chem. 70 (1998) 4836.

[22] J. de Zeeuw, R.C.M. de Mijs, L.T. Henrich, J. Chromatogr. Sci. 25 (1987) 71.

[23] Z. Ji, R.E. Majors, E.J. Guthrie, J. Chromatogr. A 842 (1999) 115.

[24] J. Dallas, P.W. Carr, Anal. Chim. Acta 251 (1982) 81.

[25] R. Atkinson, Atmos. Environ. 34 (2000) 2063.

[26] T.B. Ryerson, M. Trainer, J.S. Holloway, D.D. Parrish, L.G. Huey, D.T. Sueper, G.J. Frost, S.G. Donnelly, S. Schauffler, E.L. Atlas, W.C. Kuster, P.D. Goldan, G. Hubler, J.F. Meagher, F.C. Fehsenfeld, Science 292 (2001) 719.

[27] H. Skov, A. Lindskog, F. Palmgren, C.S. Christensen, Atmos. Environ. 35 (Suppl.) (2001) S141. 